\title{
SEPARATION OF REAL ALGEBRAIC SETS AND THE LOJASIEWICZ EXPONENT
}

\author{
KRZYSZTOF KURDYKA AND STANISEAW SPODZIEJA \\ (Communicated by Lev Borisov)
}

\begin{abstract}
We discuss several aspects of Łojasiewicz inequalities, namely local and global versions, and the relations between the gradient inequality and regular separation of real algebraic sets. We give effective estimates for Łojasiewicz's exponents in the real and complex setting.
\end{abstract}

\section{INTRODUCTION}

Łojasiewicz's inequalities are an important tool in various branches of mathematics. Quantitative aspects, like estimates (or exact computation) of Łojasiewicz exponents, are subjects of intensive study in real and complex algebraic geometry (see for instance our bibliography). Our main goal is to obtain effective estimates for the local and global Łojasiewicz exponent of an arbitrary real polynomial map. In the case of isolated zeros, effective estimates were obtained by Gwoździewicz [Gw and Kollar [K2]; see our Subsection 0.2.

In the Introduction we recall and discuss known results on various versions of Łojasiewicz's inequalities. Then we prove estimates for the length of trajectories of the gradient of a real polynomial. Using the result of D'Acunto and Kurdyka DK] on the estimate of Łojasiewicz's exponent in the local gradient inequality, we give effective estimates of the local and global Łojasiewicz exponent of an arbitrary real polynomial. Finally we obtain global effective regular separation of real algebraic sets and a generalization of the Kollár inequality.

0.1. Łojasiewicz's inequality for real maps. By $F:\left(\mathbb{R}^{n}, a\right) \rightarrow\left(\mathbb{R}^{m}, 0\right)$, where $a \in \mathbb{R}^{n}$, we denote a mapping from a neighbourhood $U \subset \mathbb{R}^{n}$ of the point $a$ to $\mathbb{R}^{m}$ such that $F(a)=0$. We put $V(F)=\{x \in U: F(x)=0\}$.

If $F:\left(\mathbb{R}^{n}, a\right) \rightarrow\left(\mathbb{R}^{m}, 0\right)$ is a real analytic mapping, then there are positive constants $C, \eta, \varepsilon$ such that the following Eojasiewicz inequality holds:

$$
|F(x)| \geq C \operatorname{dist}(x, V(F))^{\eta} \quad \text { if } \quad|x-a|<\varepsilon,
$$

where $|\cdot|$ is the Euclidean norm in $\mathbb{R}^{n}$ and $\operatorname{dist}(x, V)$ is the distance of $x \in \mathbb{R}^{n}$ to the set $V(\operatorname{dist}(x, V)=1$ if $V=\emptyset)$. The smallest exponent $\eta$ in (11) is called the Eojasiewicz exponent of $F$ at $a$ and is denoted by $\mathcal{L}_{a}(F)$. It is known that $\mathcal{L}_{a}(F)$ is a rational number and (1) holds with any $\eta \geq \mathcal{L}_{a}(F)$ and some $C, \varepsilon>0$. The

Received by the editors June 11, 2012 and, in revised form, September 14, 2012.

2010 Mathematics Subject Classification. Primary 32S50, 14B05, 34A26.

Key words and phrases. Łojasiewicz exponent, Łojasiewicz inequality with gradient, polynomial, polynomial mapping. 
exponent $\mathcal{L}_{a}(F)$ is an important invariant and tool in singularity theory (see for

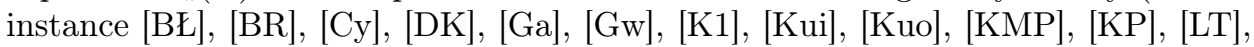
[Pł2], [RS2], [Sp], [T] $)$.

0.2. Eojasiewicz's gradient inequality. Let $f:\left(\mathbb{R}^{n}, a\right) \rightarrow(\mathbb{R}, 0)$ be a real analytic function in $x=\left(x_{1}, \ldots, x_{n}\right)$, and let $\nabla f$ be the gradient of $f$, i.e. $\nabla f=$ $\left(\frac{\partial f}{\partial x_{1}}, \ldots, \frac{\partial f}{\partial x_{n}}\right):\left(\mathbb{R}^{n}, a\right) \rightarrow \mathbb{R}^{n}$. Then there are positive constants $C, \varepsilon$ and a constant $\varrho \in[0,1)$ such that the following Lojasiewicz gradient inequality holds (cf. Ł1] or Ł2]):

$$
|\nabla f(x)| \geq C|f(x)|^{\varrho} \quad \text { if } \quad|x-a|<\varepsilon .
$$

The smallest exponent $\varrho$ in $(€)$, denoted by $\varrho_{a}(f)$, is called the Eojasiewicz exponent in the gradient inequality. The number $\varrho_{a}(f)$ is rational and ( $\mathbb{E}$ ) holds with any exponent $\varrho \geq \varrho_{a}(f)$ and some positive constants $C, \varepsilon$ (cf. [BR], [Sp]). The inequality $(\mathbb{E})$ is strongly related to the gradient conjecture of R. Thom (see [KMP]). The inequality (比) also holds for the holomorphic function $f:\left(\mathbb{C}^{n}, a\right) \rightarrow(\mathbb{C}, 0)$; see [LT.

In the case of an analytic function $f:\left(\mathbb{R}^{n}, a\right) \rightarrow(\mathbb{R}, 0)$ such that $V(f)=\{a\}$, J. Gwoździewicz [Gw] (cf. [T] for complex and [Ph] for subanalytic functions) proved that

$$
\mathcal{L}_{a}(f)=\frac{1}{1-\varrho_{a}(f)}=\mathcal{L}_{a}(\nabla f)+1
$$

The above result is not true in the general case, even if we assume that $f$ has an isolated singularity (see Remark 3).

The first aim of this paper is to show a relation between these exponents in the general case, i.e. without any assumptions about the set of zeros of $f$. Namely, for an arbitrary analytic function $f:\left(\mathbb{R}^{n}, a\right) \rightarrow(\mathbb{R}, 0)$ we prove that

$$
\mathcal{L}_{a}(f) \leq \frac{1}{1-\varrho_{a}(f)}
$$

and there are positive constants $C, \varepsilon$ such that

$$
|\nabla f(x)| \geq C \operatorname{dist}(x, V(f))^{\varrho_{a}(f) /\left(1-\varrho_{a}(f)\right)} \quad \text { if } \quad|x-a|<\varepsilon
$$

(see Corollary 1 and Corollary 2 in the complex case). If additionally $f$ has an isolated singularity at $a$, in Corollary 3 we prove that

$$
\frac{1}{1-\varrho_{a}(f)} \leq \mathcal{L}_{a}(\nabla f)+1
$$

By Gwoździewicz's result (G1), the estimates (2) and (4) are exact in terms of $\varrho_{a}(f)$. As is shown in Remark 3, we cannot expect equalities in the above estimates. It is worth noting that (3) is not directly related to the Eojasiewicz exponent $\mathcal{L}_{a}(\nabla f)$, because for $x$ close to $a$ one can have $\operatorname{dist}(x, V(f)) \ll \operatorname{dist}(x, V(\nabla f))$. The above inequalities (2) and (3) are corollaries of Theorem 11 in Section 1, which states that for a global trajectory $\gamma:[0, s) \rightarrow \mathbb{R}^{n} \backslash V(f)$ of the normed gradient vector field of $f$ (see (5)) we have

$$
\operatorname{dist}(\gamma(0), V(f)) \leq \text { length } \gamma \leq C|f(\gamma(0))|^{1-\varrho_{a}(f)}
$$

for some positive constant $C$, provided $|\gamma(0)-a|$ is small enough (cf. KMP ).

In the second part of this paper we give effective estimates of the local and global Łojasiewicz exponents for a polynomial in terms of its degree. First we shall recall 
some relevant facts. In the case of a polynomial function $f:\left(\mathbb{R}^{n}, a\right) \rightarrow(\mathbb{R}, 0)$ of degree $d$ such that $a$ is an isolated point of $V(f)$, J. Gwoździewicz $\mathrm{Gw}$ proved that $\mathcal{L}_{a}(f) \leq(d-1)^{n}+1$, so

$$
\varrho_{a}(f) \leq 1-\frac{1}{(d-1)^{n}+1}
$$

In the general case, i.e. without the assumption that $a$ is an isolated point of $V(f)$, D. D'Acunto and K. Kurdyka showed in [DK] (see also cf. [Ga])

$$
\varrho_{a}(f) \leq 1-\frac{1}{d(3 d-3)^{n-1}} \quad \text { for } \quad d \geq 2,
$$

provided $\nabla f(0)=0$. From (D-K) there follows an effective Łojasiewicz gradient inequality for a complex polynomial (see Corollary 7).

For a polynomial mapping $F=\left(f_{1}, \ldots, f_{m}\right):\left(\mathbb{R}^{n}, a\right) \rightarrow\left(\mathbb{R}^{m}, 0\right)$ with an isolated zero at $a \in \mathbb{R}^{n}$, from (G2) it follows that $\mathcal{L}_{a}(F) \leq \frac{1}{2}\left[(2 d-1)^{n}+1\right]$, where $\operatorname{deg} f_{i} \leq d$ for $i=1, \ldots, m$. In this case J. Kollár $\left[\mathrm{K} 2\right.$ proved that $\mathcal{L}_{a}(F) \leq$ $d^{n} \max \left\{\left(\begin{array}{c}n-1 \\ k\end{array}\right): 0 \leq k \leq n-1\right\}$. From Theorem 1 and (D-K) for an arbitrary polynomial function $f: \mathbb{R}^{n} \rightarrow \mathbb{R}$ of degree $d=\operatorname{deg} f \geq 2$ we obtain $\mathcal{L}_{a}(f) \leq d(3 d-3)^{n-1}$ (see Corollary 5) and $\mathcal{L}_{a}(\nabla f) \leq(d-1)(6 d-9)^{n-1}$ (see Remark 44). For any polynomial mapping $F: \mathbb{R}^{n} \rightarrow \mathbb{R}^{m}$ of degree $d$ we have

$$
\mathcal{L}_{a}(F) \leq d(6 d-3)^{n-1}
$$

for $a \in \mathbb{R}^{n}$ (see Corollary [6). The above result holds without any assumption on the set $V(F)$.

0.3. Regular separation of algebraic sets. We also obtain an effective estimate for the Eojasiewicz exponent in the separation inequality for real algebraic sets (see Corollary 8). More precisely, for polynomial mappings $g, h: \mathbb{R}^{n} \rightarrow \mathbb{R}^{m}$ and algebraic sets $X=V(g), Y=V(h)$ for any $a \in X \cap Y$ there exists a positive constant $C$ such that

$$
\operatorname{dist}(x, X)+\operatorname{dist}(x, Y) \geq C \operatorname{dist}(x, X \cap Y)^{d(6 d-3)^{n-1}}
$$

in a neighbourhood of $a$, where $d=\max \{\operatorname{deg} g, \operatorname{deg} h\}$. A similar result in the complex case was obtained in $[\mathrm{Cy}], \mathrm{CKT}],[\mathrm{JKS}]$. In Corollary 9 we apply the above estimate for the separation of a complex algebraic set $V \subset \mathbb{C}^{n}$ from $\mathbb{R}^{n} \subset \mathbb{C}^{n}$.

For complex algebraic sets $X, Y \subset \mathbb{C}^{n}$ E. Cygan in $[\mathrm{Cy}$, Theorem 4.6] (cf. $[\mathrm{Br}$, [JKS], [K1]) proved the following global Łojasiewicz inequality:

$$
\operatorname{dist}(z, X)+\operatorname{dist}(z, Y) \geq C\left(\frac{\operatorname{dist}(z, X \cap Y)}{1+|z|^{2}}\right)^{\operatorname{deg} X \cdot \operatorname{deg} Y} \quad \text { for } \quad z \in \mathbb{C}^{n},
$$

where $C$ is a positive constant. This inequality is strongly related to the effective Nullstellensatz (see [K1]). It is also a useful tool in extending regular mappings with preservation of the Łojasiewicz exponent (see [O]). By using the method of Cygan Cy and Corollary 8 we prove the following real version of the above inequality (see Theorem 2): if $g, h: \mathbb{R}^{n} \rightarrow \mathbb{R}^{m}$ are polynomial mappings, $X=V(g), Y=V(h)$ and $d=\max \{\operatorname{deg} g, \operatorname{deg} h\}$, then for some positive constant $C$,

$$
\operatorname{dist}(x, X)+\operatorname{dist}(x, Y) \geq C\left(\frac{\operatorname{dist}(x, X \cap Y)}{1+|x|^{2}}\right)^{d(6 d-3)^{n-1}} \quad \text { for } \quad x \in \mathbb{R}^{n} .
$$


In particular, for a polynomial mapping $F: \mathbb{R}^{n} \rightarrow \mathbb{R}^{m}$ of degree $d$ there exists a positive constant $C$ such that

$$
|F(x)| \geq C\left(\frac{\operatorname{dist}(x, V(F))}{1+|x|^{2}}\right)^{d(6 d-3)^{n-1}} \quad \text { for } \quad x \in \mathbb{R}^{n}
$$

(see Corollary [10, cf. [Br], [JKS], [K1], [Cy, Theorem 5.1]). If additionally the set $V(F)$ is compact, the Łojasiewicz exponent of $F$ at infinity (denoted by $\mathcal{L}_{\infty}(F)$, see Section (4) is estimated from below by $-d(6 d-3)^{n-1}$; i.e. for some positive constants $C, R$,

$$
|F(x)| \geq C|x|^{-d(6 d-3)^{n-1}} \quad \text { for } \quad x \in \mathbb{R}^{n}, \quad|x| \geq R
$$

(see Corollary [11). The Łojasiewicz exponent at infinity of a mapping has been considered by many authors in the context of effective Nullstellensatz and properness of mappings (see for instance [Bi], [Br], [C], [CK1], [CK2], $\mathrm{Cy}$, [CKT], GS], [Ha, [J1], JJ2, [JK], JKS], [K1], [Pa], [Pł1], [RS1], [RS3], [Sk], [Sp]).

In the complex domain the most deep estimation of the exponent $\mathcal{L}_{\infty}(F)$ for a polynomial mapping $F=\left(f_{1}, \ldots, f_{m}\right): \mathbb{C}^{n} \rightarrow \mathbb{C}^{m}$ has been given by J. Kollár [K1]. Namely, if $F$ has a finite number of zeroes and $d_{j}=\operatorname{deg} f_{j}, d_{1} \geq \ldots \geq d_{m}>2$, then

$$
\mathcal{L}_{\infty}(F) \geq d_{m}-d_{1} \cdots d_{n},
$$

provided $m \geq n$. Corollary 11 is a generalization of the above estimation to the case of real polynomial mappings with a compact set of real zeroes.

In these estimates the exponent $p=d(6 d-3)^{n-1}$ probably is not sharp; however, asymptotically this bound is of order $O\left(d^{n}\right)$ as Example 1 shows.

These two global inequalities can also be interpreted as a parametric version of the local separation of real algebraic sets ( $\mathrm{KS}$ ). What is interesting (and new to our knowledge) is that we give an estimate for the asymptotics of the constant $C$. Namely in a ball $\bar{B}(a, 1)$ the inequality $(\overline{\mathrm{KS}})$ holds with a constant $C(a)=\left(\frac{C}{1+(1+|a|)^{2}}\right)^{d(6 d-3)^{n-1}}$, where $C$ does not depend on $a$.

\section{LENGTH OF TRAJECTORY}

Let $f:\left(\mathbb{R}^{n}, a\right) \rightarrow(\mathbb{R}, 0), a \in \mathbb{R}^{n}$, be a nonzero analytic function, let $\varrho \in(0,1)$ and $C>0$ be such that the inequality ( $(\mathbb{E})$ holds in a neighbourhood $U \subset \mathbb{R}^{n}$ of the point $a$, and let $V=V(f)$. Let $\varphi(t)=|t|^{1-\varrho}$ for $t \in \mathbb{R}$. We have (cf. [Kur], [KMP])

Proposition 1 (Kurdyka-Łojasiewicz inequality). Under the above notation,

$$
|\nabla(\varphi \circ f)(x)| \geq(1-\varrho) C \quad \text { for } x \in U \backslash V .
$$

Proof. Since $\varphi^{\prime}(t)=(1-\varrho)|t|^{-\varrho} \operatorname{sign} t$ for $t \neq 0$, we have

$$
\varphi^{\prime}(f(x))=(1-\varrho)|f(x)|^{-\varrho} \operatorname{sign} f(x) \quad \text { for } x \in U \backslash V .
$$

By (迆) we have $|f(x)|^{-\varrho}|\nabla f(x)| \geq C$ for $x \in U \backslash V$. Since $\nabla(\varphi \circ f)=\left(\varphi^{\prime} \circ f\right) \nabla f$, the above inequality yields

$$
|\nabla(\varphi \circ f)(x)|=(1-\varrho)|f(x)|^{-\varrho}|\nabla f(x)| \geq(1-\varrho) C \quad \text { for } x \in U \backslash V .
$$

This gives the assertion. 
Remark 1. In [Kur] a generalization of Łojasiewicz's gradient inequality to ominimal structures was given. In this generalization the function $\varphi$ is definable. This result was subsequently generalized to a more general situation in [BDLM], where the name Kurdyka-Łojasiewicz inequality was introduced.

Since the inequality ( $\mathbb{E}$ ) holds in $U$, the vector field $\nabla f$ is nonzero in $U \backslash V$. So

$$
H(x)=-\operatorname{sign} f(x) \frac{\nabla f(x)}{|\nabla f(x)|} \quad \text { for } x \in U \backslash V
$$

is well defined and locally Lipschitz. Let $\gamma:[0, s) \rightarrow U \backslash V$ be a maximal solution (to the right) of the equation

$$
x^{\prime}=H(x) .
$$

Then the function $f \circ \gamma$ is decreasing if $f(\gamma(0))>0$ and increasing if $f(\gamma(0))<0$. Hence $\lim _{s_{1} \rightarrow s} f \circ \gamma\left(s_{1}\right)$ exists. Since $\left|\gamma^{\prime}(t)\right|=1$, we have length $\gamma=s$.

Theorem 1. If $\gamma(0)$ is sufficiently close to $a$, then

$$
\operatorname{dist}(\gamma(0), V(f)) \leq \operatorname{length} \gamma \leq \frac{1}{(1-\varrho) C}|f(\gamma(0))|^{1-\varrho} .
$$

Proof. Since $\frac{\nabla f(x)}{|\nabla f(x)|} \operatorname{sign} f(x)=\frac{\nabla(\varphi \circ f)(x)}{|\nabla(\varphi \circ f)(x)|} \quad$ for $x \in U \backslash V$, it follows that

$$
\begin{aligned}
(\varphi \circ f \circ \gamma)^{\prime} & =\left\langle\nabla(\varphi \circ f) \circ \gamma, \gamma^{\prime}\right\rangle=-\left\langle\nabla(\varphi \circ f) \circ \gamma, \frac{\nabla(\varphi \circ f) \circ \gamma}{|\nabla(\varphi \circ f) \circ \gamma|}\right\rangle \\
& =-|\nabla(\varphi \circ f) \circ \gamma|,
\end{aligned}
$$

where $\langle\cdot, \cdot\rangle$ is the standard scalar product in $\mathbb{R}^{n}$. Let $s_{1} \in[0, s)$ and $\gamma_{s_{1}}=\left.\gamma\right|_{\left[0, s_{1}\right]}$. Then length $\gamma_{s_{1}}=s_{1}$. From the above and Proposition 1 we see that

$$
\begin{aligned}
\varphi(f(\gamma(0)))-\varphi\left(f\left(\gamma\left(s_{1}\right)\right)\right) & =(\varphi \circ f \circ \gamma)^{\prime}(t)\left(-s_{1}\right)=-|\nabla(\varphi \circ f) \circ \gamma(t)|\left(-s_{1}\right) \\
& \geq(1-\varrho) C s_{1}=(1-\varrho) C \text { length } \gamma_{s_{1}}
\end{aligned}
$$

for some $t \in\left[0, s_{1}\right]$. Consequently,

$$
\text { length } \gamma_{s_{1}} \leq \frac{1}{(1-\varrho) C}\left[|f(\gamma(0))|^{1-\varrho}-\left|f\left(\gamma\left(s_{1}\right)\right)\right|^{1-\varrho}\right] .
$$

Letting $s_{1} \rightarrow s$ we have $\left|f\left(\gamma\left(s_{1}\right)\right)\right|^{1-\varrho} \rightarrow \alpha$, where $\alpha \geq 0$, and so

$$
\text { length } \gamma \leq \frac{1}{(1-\varrho) C}\left(|f(\gamma(0))|^{1-\varrho}-\alpha\right) \leq \frac{1}{(1-\varrho) C}|f(\gamma(0))|^{1-\varrho} .
$$

Let $r>0$ be such that the ball centred at $a$ of radius $r$ is contained in $U$. Let $0<r^{\prime}<\frac{r}{2}$ be such that if $|x-a| \leq r^{\prime}$, then $\frac{1}{(1-\varrho) C}|f(x)|^{1-\varrho} \leq \frac{r}{2}$.

Assume now that $|\gamma(0)-a|<r^{\prime}$. Then by (6) and the above, length $\gamma \leq$ $\frac{r}{2}$, so $\lim _{t \rightarrow s} \gamma(t)$ certainly exists. Hence $\lim _{t \rightarrow s} \gamma(t) \in V$, because length $\gamma<$ $\operatorname{dist}\left(\gamma(0), \mathbb{R}^{n} \backslash U\right)$. In consequence, length $\gamma \geq \operatorname{dist}(\gamma(0), V(f))$. This, together with (6), gives the assertion.

Remark 2. Historically this type of argument already appeared in the original paper of Łojasiewicz [ $\mathrm{E}$, and then in many other papers, e.g. [BM], Ga], [KMP].

Corollary 1. Under the assumptions and notation of Theorem 1 in a neighbourhood of a we have

$$
|f(x)| \geq C_{1} \operatorname{dist}(x, V)^{1 /(1-\varrho)},
$$


with $C_{1}=(C(1-\varrho))^{1 /(1-\varrho)}$, in particular $\mathcal{L}_{a}(f) \leq 1 /(1-\varrho)$, and

$$
|\nabla f(x)| \geq C_{2} \operatorname{dist}(x, V)^{\varrho /(1-\varrho)},
$$

with $C_{2}=C C_{1}^{\varrho}=C(C(1-\varrho))^{\varrho /(1-\varrho)}$.

Proof. The inequality (7) follows immediately from Theorem 17. From (17) and (飞) we obtain (8).

Interestingly, inequalities in Corollary 1 are satisfied in the complex case. Namely, we have

Corollary 2. Let $a \in \mathbb{C}^{n}$ and let $f:\left(\mathbb{C}^{n}, a\right) \rightarrow(\mathbb{C}, 0)$ be a holomorphic function. Let $\varrho \in[0,1)$ and $C, \varepsilon>0$ be constants for which the Eojasiewicz gradient inequality (区) holds. Then inequalities (77) and (8) are satisfied in a neighbourhood of a.

Proof. Let $g=|f|^{2}:\left(\mathbb{R}^{2 n}: a\right) \rightarrow(\mathbb{R}, 0)$. Since $f$ is a holomorphic function, by Cauchy-Riemann equations we obtain $|\nabla g|=2|f||\nabla f|$. Hence, by (飞),

$$
|\nabla g(x)| \geq 2 C|g(x)|^{(\varrho+1) / 2} \quad \text { in a neighbourhood of } a .
$$

If $x$ is sufficiently close to $a$, from (9) and Corollary 1 we obtain

$$
\operatorname{dist}(x, V(f))=\operatorname{dist}(x, V(g)) \leq \frac{1}{(1-\varrho) C}|g(x)|^{(1-\varrho) / 2}=\frac{1}{(1-\varrho) C}|f(x)|^{1-\varrho},
$$

which proves (7). Hence (飞) gives (8).

\section{2. ŁOJASIEWICZ EXPONENT OF AN ANALYTIC FUNCTION AT AN ISOLATED SINGULARITY}

Let $f:\left(\mathbb{R}^{n}, a\right) \rightarrow(\mathbb{R}, 0), a \in \mathbb{R}^{n}$, be a nonzero analytic function. If $f$ has an isolated singularity at $a$, i.e. $a$ is an isolated zero of $\nabla f(x)$, we have

Corollary 3. If $f$ has an isolated singularity at $a$, then

$$
\mathcal{L}_{a}(f) \leq \frac{1}{1-\varrho_{a}(f)} \leq \mathcal{L}_{a}(\nabla f)+1
$$

Proof. The inequality $\mathcal{L}_{a}(f) \leq \frac{1}{1-\varrho_{a}(f)}$ follows immediately from Corollary 1 We prove the right inequality of (10). For simplicity of notation we assume that $a=0$ and put $\varrho=\varrho_{0}(f)$. Let $U \subset \mathbb{R}^{n}$ be a neighbourhood of the origin and let $C$ be a positive constant such that

$$
|\nabla f(x)| \geq C|f(x)|^{\varrho} \quad \text { for } \quad x \in U .
$$

By Theorem 1.4 in $\left[\mathrm{Sp}\right.$, there exist a positive constant $C^{\prime}$ and an analytic curve $\gamma:[0, \varepsilon) \rightarrow U$, where $\varepsilon>0, f(\gamma(0))=0$, and $\gamma(t) \neq 0$ for $t \in(0, \varepsilon)$, such that

$$
|\nabla f(\gamma(t))| \leq C^{\prime}|f(\gamma(t))|^{\varrho} \quad \text { for } \quad t \in[0, \varepsilon) .
$$

Since 0 is an isolated singularity of $f$, we have $\gamma(0)=0$ and we may assume that $f(\gamma(t)) \neq 0$ for $t \in(0, \varepsilon)$. Then

$$
\frac{1}{\varrho} \operatorname{ord} \nabla f \circ \gamma \geq \operatorname{ord} f \circ \gamma \geq \operatorname{ord} \nabla f \circ \gamma+\text { ord } \gamma,
$$

so decreasing $\varepsilon$ if necessary, for some $C^{\prime \prime}>0$,

$$
|\nabla f(\gamma(t))|^{(1-\varrho) / \varrho} \leq C^{\prime \prime}|\gamma(t)| \text { for } t \in[0, \varepsilon) .
$$

Consequently, $\frac{\varrho}{1-\varrho} \leq \mathcal{L}_{0}(\nabla f)$. This gives the right inequality of (10) and ends the proof. 
Remark 3. We cannot demand equalities in (10). Indeed, for the Kuo example (see Gw]) of the polynomial $k(x, y)=x^{3}+3 x y^{4}$ we have $\mathcal{L}_{0}(k)=3$ and $\varrho_{0}(k)=2 / 3$ and $\mathcal{L}_{0}(\nabla k)=4$, so

$$
\mathcal{L}_{0}(k)=\frac{1}{1-\varrho_{0}(k)}<\mathcal{L}_{0}(\nabla k)+1
$$

For the polynomial $g(x, y)=y^{2}-x^{4}$ we have $\mathcal{L}_{0}(g)=2$ and $\varrho_{0}(g)=3 / 4$ and $\mathcal{L}_{0}(\nabla g)=3$, so

$$
\mathcal{L}_{0}(g)<\frac{1}{1-\varrho_{0}(g)}=\mathcal{L}_{0}(\nabla g)+1 .
$$

For the polynomial $h(x, y)=y^{2}-x^{3}$ equalities hold in (10). Note that $k, g$ and $h$ have isolated singularities at the origin.

\section{Effective estimates of the ŁojasiewicZ EXPONEnT}

From (G2), (D-K) and Theorem 1 we obtain

Corollary 4. Let $f:\left(\mathbb{R}^{n}, a\right) \rightarrow(\mathbb{R}, 0)$, where $a \in \mathbb{R}^{n}$, be a polynomial function of degree $\operatorname{deg} f \geq 2$, and let $d \in \mathbb{N}, d \geq \operatorname{deg} f$. Then there exist a neighbourhood $U \subset \mathbb{R}^{n}$ of $a$ and a positive constant $C$ such that for any global trajectory $\gamma$ : $[0, s) \rightarrow U \backslash V(f)$ of the vector field (5),

$$
\text { length } \gamma \leq C|f(\gamma(0))|^{\frac{1}{d(3 d-3)^{n-1}}} .
$$

If additionally $a$ is an isolated zero of $f$, then

$$
\text { length } \gamma \leq C|f(\gamma(0))|^{\frac{1}{(d-1)^{n}+1}} .
$$

Proof. There exists a neighbourhood $U \subset \mathbb{R}^{n}$ of $a$ such that (E) holds for $\varrho=$ $1-\frac{1}{d(3 d-3)^{n-1}}$. If $a$ is an isolated zero of $f$, then $(\mathbb{E})$ holds for $\varrho=1-\frac{1}{(d-1)^{n}+1}$. Then Theorem 1 yields the assertion.

From Corollary 4 and Theorem 1 we easily obtain the Gwoździewicz result $\mathrm{Gw}$, Theorem 1.5]: $\mathcal{L}_{0}(f) \leq(\operatorname{deg} f-1)^{n-1}+1$ for a polynomial function $f:\left(\mathbb{R}^{n}, 0\right) \rightarrow$ $(\mathbb{R}, 0)$ having an isolated zero at 0 . In the general case we have

Corollary 5. For any polynomial function $f: \mathbb{R}^{n} \rightarrow \mathbb{R}$ of degree $\operatorname{deg} f \geq 2$, any $d \in \mathbb{N}, d \geq \operatorname{deg} f$, and any $a \in \mathbb{R}^{n}$ we have $\mathcal{L}_{a}(f) \leq d(3 d-3)^{n-1}$.

Proof. If $a \notin V(f)$, then the assertion is obvious. In the case $a \in V(f)$ the assertion follows immediately from Corollary 4 and Theorem 1 .

Corollary 6. Let $F=\left(f_{1}, \ldots, f_{m}\right): \mathbb{R}^{n} \rightarrow \mathbb{R}^{m}$ be a polynomial mapping of degree $d$, and let $a \in \mathbb{R}^{n}$. Then $\mathcal{L}_{0}(F) \leq d(6 d-3)^{n-1}$.

Proof. It suffices to apply Corollary 5 for $f=f_{1}^{2}+\cdots+f_{m}^{2}$.

Remark 4. For a polynomial function $f: \mathbb{R}^{n} \rightarrow \mathbb{R}$ of degree $\operatorname{deg} f=d \geq 2$, the above corollary gives $\mathcal{L}_{a}(\nabla f) \leq(d-1)(6 d-9)^{n-1}$.

Example 1. J. Kollar [K2 gave the following example (which is a modification of an example of Masser, Philippon, and Brownawell $[\mathrm{Br}])$. Set $f_{1}=x_{1}^{d}$ and $f_{i}=$ $x_{i-1}-x_{i}^{d}$ for $i=2, \ldots, n$. Then $\Phi(x):=\max _{i}\{|f i(x)|\}>0$ for $x \neq 0$. Let $p(t)=\left(t^{d^{n-1}}, t^{d^{n-2}}, \ldots, t\right)$. Then $\lim _{t \rightarrow 0}\|p(t)\| /|t|=1$ and $\Phi(p(t))=t^{d^{n}}$. Set $F=\left(f_{1}, \ldots, f_{m}\right): \mathbb{R}^{n} \rightarrow \mathbb{R}^{n}$. 
Thus $\mathcal{L}_{0}(F) \leq d^{n}$; in fact there is equality. This holds both over $\mathbb{R}$ and $\mathbb{C}$. In the real case set $f=\sum f_{i}^{2}$. Then $\operatorname{deg} f=2 d, f$ has an isolated real zero at the origin and $\mathcal{L}_{0}(f)=2 d^{n}$.

From (D-K) and (9) we immediately obtain the following effective Łojasiewicz gradient inequality for complex polynomials.

Corollary 7. Let $f: \mathbb{C}^{n} \rightarrow \mathbb{C}$ be a polynomial of degree $d \geq 2$ with $f(0)=0$, and let $\varrho=1-\frac{1}{d(6 d-3)^{2 n-1}}$. Then there are $C, \varepsilon>0$ such that

$$
|\nabla f(z)| \geq C|f(z)|^{\varrho} \quad \text { for } \quad z \in \mathbb{C}^{n}, \quad|z|<\varepsilon .
$$

From Corollary 5 we obtain an effective Łojasiewicz separation inequality.

Corollary 8. Let $X=V\left(g_{1}, \ldots, g_{k}\right)$ and $Y=V\left(h_{1}, \ldots, h_{l}\right) \subsetneq \mathbb{R}^{n}$, where $g_{i}, h_{j}$ : $\mathbb{R}^{n} \rightarrow \mathbb{R}$ are polynomials of degree not greater than $d$. Let $a \in \mathbb{R}^{n}$. Then there exists a positive constant $C$ such that

$$
\operatorname{dist}(x, X)+\operatorname{dist}(x, Y) \geq C \operatorname{dist}(x, X \cap Y)^{d(6 d-3)^{n-1}}
$$

in a neighbourhood of a. If additionally $a$ is an isolated point of $X \cap Y$, then

$$
\operatorname{dist}(x, X)+\operatorname{dist}(x, Y) \geq C \operatorname{dist}(x, X \cap Y)^{\frac{(2 d-1)^{n}+1}{2}}
$$

in a neighbourhood of a.

Proof. We prove (13). If $a \notin X \cap Y$, then the assertion is obvious. Assume that $a \in X \cap Y$. It suffices to prove (13) for $x \in X$ (see [11, p. 61]). If $X \subset Y$, then the inequality holds with $\varrho=1$, so the assertion holds. Assume that $X \not \subset Y$. Let $f=g_{1}^{2}+\cdots+g_{k}^{2}+h_{1}^{2}+\cdots+h_{l}^{2}$. Then $2 d \geq 2 \operatorname{deg} f \geq 2$ and $V(f)=X \cap Y$. So by Corollary 5 there exist positive constants $C, C^{\prime}$ and a neighbourhood $U \subset \mathbb{R}^{n}$ of $a$ such that for any $x \in U \cap(X \backslash Y)$,

$$
\operatorname{dist}(x, X \cap Y)^{\frac{2 d(6 d-3)^{n-1}}{2}} \leq C^{\prime}|f(x)|^{\frac{1}{2}}=C^{\prime}\left|\left(h_{1}(x), \ldots, h_{l}(x)\right)\right| \leq C \operatorname{dist}(x, Y) .
$$

This gives (13) (cf. Claim 1 in the proof of Theorem 2 in Section 44). The inequality (14) is proved analogously.

Corollary 8 yields an effective Łojasiewicz inequality for the separation of a complex algebraic set from $\mathbb{R}^{n}$.

Corollary 9. Let $f:\left(\mathbb{C}^{n}, 0\right) \rightarrow\left(\mathbb{C}^{m}, 0\right)$ be a polynomial mapping of degree $d \geq 1$ and let $V_{\mathbb{C}}=\left\{z \in \mathbb{C}^{n}: f(z)=0\right\}, V_{\mathbb{R}}=\left\{x \in \mathbb{R}^{n}: f(x)=0\right\}$. Then there exists $C>0$ such that

$$
\operatorname{dist}\left(z, V_{\mathbb{C}}\right)+\operatorname{dist}\left(z, \mathbb{R}^{n}\right) \geq C \operatorname{dist}\left(z, V_{\mathbb{R}}\right)^{d(6 d-3)^{2 n-1}}
$$

in a neighbourhood of the origin of $\mathbb{C}^{n}$.

Proof. Let $g=\left(g_{1}, \ldots, g_{2 m}\right):\left(\mathbb{R}^{2 n}, 0\right) \rightarrow\left(\mathbb{R}^{2 m}, 0\right)$ be of the form

$$
g=\left(\operatorname{Re} f_{1}, \operatorname{Im} f_{1}, \ldots, \operatorname{Re} f_{m}, \operatorname{Im} f_{m}\right),
$$

and $h_{i}\left(x_{1}, y_{1}, \ldots, x_{m}, y_{m}\right)=y_{i}$ for $i=1, \ldots, m$. Then $V_{\mathbb{C}}=g^{-1}(0)$ and $V_{\mathbb{C}} \cap \mathbb{R}^{n}=$ $V_{\mathbb{R}}=V\left(h_{1}, \ldots, h_{m}\right)$. So, Corollary 8 gives the assertion. 


\section{Global Łojasiewicz inequality}

We prove the following real version of the global Łojasiewicz inequality (C):

Theorem 2. Let $X, Y \subsetneq \mathbb{R}^{n}$ be algebraic sets, and let $g_{1}, \ldots, g_{k}, h_{1}, \ldots, h_{l}: \mathbb{R}^{n} \rightarrow$ $\mathbb{R}$ be polynomials such that $X=V\left(g_{1}, \ldots, g_{k}\right)$ and $Y=V\left(h_{1}, \ldots, h_{l}\right)$. Let $d=$ $\max \left\{\operatorname{deg} g_{1}, \ldots, \operatorname{deg} g_{k}, \operatorname{deg} h_{1}, \ldots, \operatorname{deg} h_{l}\right\}$. Then there exists a positive constant $C$ such that

$$
\operatorname{dist}(x, X)+\operatorname{dist}(x, Y) \geq C\left(\frac{\operatorname{dist}(x, X \cap Y)}{1+|x|^{2}}\right)^{d(6 d-3)^{n-1}} \quad \text { for } \quad x \in \mathbb{R}^{n} .
$$

Proof. Let $p=d(6 d-3)^{n-1}$. If $X=\emptyset$ or $Y=\emptyset$, then the assertion is obvious. Assume that $X \neq \emptyset$ and $Y \neq \emptyset$.

Claim 1. Assertion (15) is equivalent to

$$
\operatorname{dist}(x, Y) \geq C^{\prime}\left(\frac{\operatorname{dist}(x, X \cap Y)}{1+|x|^{2}}\right)^{p} \quad \text { for } \quad x \in X
$$

for some positive constant $C^{\prime}$ (cf. [Cy, Lemma 4.2] and [ [11, p. 61]). The implication (15) $\Rightarrow$ (16) is obvious. Assume that (15) fails. Then there exists a sequence $\left(a_{\nu}\right)$ such that

$$
\operatorname{dist}\left(a_{\nu}, X\right)+\operatorname{dist}\left(a_{\nu}, Y\right)<\frac{1}{\nu}\left(\frac{\operatorname{dist}\left(a_{\nu}, X \cap Y\right)}{1+\left|a_{\nu}\right|^{2}}\right)^{p} \quad \text { for } \quad \nu \in \mathbb{N} .
$$

Let $b_{\nu} \in X$ and $c_{\nu} \in Y$ be such that

$$
d\left(a_{\nu}, X\right)=\left|a_{\nu}-b_{\nu}\right|, \quad d\left(a_{\nu}, Y\right)=\left|a_{\nu}-c_{\nu}\right| .
$$

Then

$$
d\left(a_{\nu}, X\right)+d\left(a_{\nu}, Y\right)=\left|a_{\nu}-c_{\nu}\right|+\left|a_{\nu}-b_{\nu}\right| \geq\left|c_{\nu}-b_{\nu}\right| \geq d\left(b_{\nu}, Y\right) .
$$

Hence

$$
\operatorname{dist}\left(b_{\nu}, Y\right)<\frac{1}{\nu}\left(\frac{\operatorname{dist}\left(a_{\nu}, X \cap Y\right)}{1+\left|a_{\nu}\right|^{2}}\right)^{p} \quad \text { for } \quad \nu \in \mathbb{N},
$$

which contradicts (16) and proves Claim 1.

Claim 2. One can assume that the set $Y$ is a linear subspace of $\mathbb{R}^{n}$ (cf. Cy, Lemma 4.3]). Indeed, in $\mathbb{R}^{2 n}$ we have the norm $\|(x, y)\|=|x|+|y|$ for $x \in \mathbb{R}^{n}$ and $y \in \mathbb{R}^{n}$. Then for $\Delta=\left\{(x, x) \in \mathbb{R}^{2 n}: x \in \mathbb{R}^{n}\right\}$ and $(x, x) \in \Delta$ we have $\operatorname{dist}((x, x), X \times Y)=$ $\operatorname{dist}(x, X)+\operatorname{dist}(x, Y)$ and $\operatorname{dist}((x, x), \Delta \cap(X \times Y))=2 \operatorname{dist}(x, X \cap Y)$ if $X \cap Y \neq \emptyset$ and $\operatorname{dist}((x, x), \Delta \cap(X \times Y))=1$ if $X \cap Y=\emptyset$. So, we see that (15) is equivalent to

$$
\operatorname{dist}((x, x), X \times Y) \geq C^{\prime}\left(\frac{\operatorname{dist}((x, x), \Delta \cap(X \times Y))}{1+\|(x, x)\|^{2}}\right)^{p}, \quad(x, x) \in \Delta,
$$

for a constant $C^{\prime}>0$. Moreover, $X \times Y=V\left(g_{1}(x), \ldots, g_{k}(x), h_{1}(y), \ldots, h_{l}(y)\right)$ and $\Delta=V\left(x_{1}-y_{1}, \ldots, x_{n}-y_{n}\right)$, and obviously $d=\max \left\{\operatorname{deg} g_{1}(x), \ldots, \operatorname{deg} g_{k}(x)\right.$, $\left.\operatorname{deg} h_{1}(y), \ldots, \operatorname{deg} h_{l}(y), \operatorname{deg}\left(x_{1}-y_{1}\right), \ldots, \operatorname{deg}\left(x_{n}-y_{n}\right)\right\}$. Hence, by Claim 1, it suffices to prove the assertion for $\Delta$ and $X \times Y$, and thus Claim 2 follows.

Claim 3. There exists a positive constant $C$ such that (16) holds. Indeed, according to Claims 1 and 2, we assume that $Y$ is a linear subspace of $\mathbb{R}^{n}$. Moreover, we may assume that $Y=\mathbb{R}^{j} \times\{0\}$, and $h_{1}=x_{j+1}, \ldots, h_{n-j}=x_{n}$, where $l=n-j$. 
Assume to the contrary that (16) does not hold for any $C>0$. Then there exists a sequence $\left(a_{\nu}\right), a_{\nu}=\left(a_{1, \nu}, \ldots, a_{n, \nu}\right) \in X$, such that

$$
\operatorname{dist}\left(a_{\nu}, Y\right)<\frac{1}{\nu}\left(\frac{\operatorname{dist}\left(a_{\nu}, X \cap Y\right)}{1+\left|a_{\nu}\right|^{2}}\right)^{p} \quad \text { for } \quad \nu \in \mathbb{N} \text {. }
$$

By Claim 1 and Corollary 8 we see that $\left|a_{\nu}\right| \rightarrow \infty$. So, we may assume that the sequence $\bar{a}_{\nu}=\left(1: a_{1, \nu}: \ldots: a_{n, \nu}\right) \in \mathbb{P}^{n}(\mathbb{R})$ is convergent to some $\bar{a}_{0}=(0$ : $\left.a_{1,0}: \ldots: a_{n, 0}\right) \in \mathbb{P}^{n}(\mathbb{R})$, where $\mathbb{P}^{n}(\mathbb{R})$ is the real projective space of dimension $n$. Then for some $s \in\{1, \ldots, n\}$ we have $a_{s, 0} \neq 0$, so we may assume that $a_{s, 0}=1$ and $a_{s, \nu} \neq 0$ for $\nu \in \mathbb{N}$, and, moreover, that $\left|a_{s, \nu}\right| \geq\left|a_{i, \nu}\right|$ for $i=1, \ldots, n$ and any $\nu \in \mathbb{N}$. Consequently, $\left|a_{s, \nu}\right| \rightarrow \infty$ as $\nu \rightarrow \infty$. By (17) we see that $\left|\left(a_{j+1, \nu}, \ldots, a_{n, \nu}\right)\right|=$ $\operatorname{dist}\left(a_{\nu}, Y\right) \rightarrow 0$ as $\nu \rightarrow \infty$, so we easily obtain $a_{j+1,0}=\cdots=a_{n, 0}=0$. Hence,

$$
\bar{a}_{0}=\left(0: a_{1,0}: \ldots: a_{j, 0}: 0: \ldots: 0\right) \text { and } s \in\{1, \ldots, j\} .
$$

Choosing an appropriate coordinate system we may assume that

$$
\left|a_{i, 0}\right|<\frac{1}{3} \text { for } i \neq s \text {. }
$$

The polynomials $g_{i}^{*}\left(x_{0}, \ldots, x_{n}\right)=x_{0}^{\operatorname{deg} g_{i}} g\left(\frac{x_{1}}{x_{0}}, \ldots, \frac{x_{n}}{x_{0}}\right)$ are homogeneous, $g_{i}=$ $g_{i}^{*}\left(1, x_{1}, \ldots, x_{n}\right)$ and $\operatorname{deg} g_{i}^{*}=\operatorname{deg} g_{i}$ for $i=1, \ldots, k$. Consider the sets

$$
\begin{aligned}
& \bar{X}=\left\{\bar{x} \in \mathbb{P}^{n}(\mathbb{R}): g_{1}^{*}(\bar{x})=\cdots=g_{k}^{*}(\bar{x})=0\right\}, \\
& \bar{Y}=\left\{\left(x_{0}: \ldots: x_{n}\right) \in \mathbb{P}^{n}(\mathbb{R}): x_{j+1}=\cdots=x_{n}=0\right\} .
\end{aligned}
$$

Then $\bar{a}_{\nu} \in \bar{X}$ for $\nu \in \mathbb{N}$, and by (18) we have $\bar{a}_{0} \in \bar{X} \cap \bar{Y}$.

Put $U_{s}=\left\{\left(x_{0}: \ldots: x_{n}\right) \in \mathbb{P}^{n}(\mathbb{R}): x_{s} \neq 0\right\}$. Take the chart

$$
\varphi: U_{s} \ni\left(x_{0}: \ldots: x_{n}\right) \mapsto\left(\frac{x_{0}}{x_{s}}, \ldots, \frac{x_{s-1}}{x_{s}}, \frac{x_{s+1}}{x_{s}}, \ldots, \frac{x_{n}}{x_{s}}\right) \in \mathbb{R}^{n} .
$$

Let $X_{s}=\varphi\left(\bar{X} \cap U_{s}\right), Y_{s}=\varphi\left(\bar{Y} \cap U_{s}\right)$, and $g_{i, s}=g_{i}^{*}\left(z_{0}, \ldots, z_{s-1}, 1, z_{s+1}, \ldots, z_{n}\right)$ for $i=1, \ldots, k$. Then $X_{s}=\left\{z \in \mathbb{R}^{n}: g_{1, s}(z)=\cdots=g_{k, s}(z)=0\right\}$ and $Y_{s}=\{z \in$ $\left.\mathbb{R}^{n}: z_{j+1}=\cdots=z_{n}=0\right\}$, where $z=\left(z_{0}, \ldots, z_{s-1}, z_{s+1}, \ldots, z_{n}\right) \in \mathbb{R}^{n}$. Moreover,

$$
d \geq \max \left\{\operatorname{deg} g_{1, s}, \ldots, \operatorname{deg} g_{k, s}, \operatorname{deg} z_{j+1}, \ldots, \operatorname{deg} z_{n}\right\} .
$$

Let $u_{\nu}=\varphi\left(\bar{a}_{\nu}\right)$ for $\nu \in \mathbb{N}$, and $u_{0}=\varphi\left(\bar{a}_{0}\right)$. Then $u_{\nu} \rightarrow u_{0}$ as $\nu \rightarrow \infty$.

By (20) and Corollary 8 there exists $C^{\prime \prime}>0$ such that

$$
\operatorname{dist}\left(u_{\nu}, Y_{s}\right) \geq C^{\prime \prime} \operatorname{dist}\left(u_{\nu}, X_{s} \cap Y_{s}\right)^{p} \quad \text { for sufficiently large } \quad \nu \in \mathbb{N} \text {. }
$$

Thus we may assume that the above holds for any $\nu \in \mathbb{N}$. So from (17) it follows that

$$
\begin{gathered}
\frac{1}{\nu} \frac{1}{\left|a_{\nu}\right|}\left(\frac{\operatorname{dist}\left(a_{\nu}, X \cap Y\right)}{1+\left|a_{\nu}\right|^{2}}\right)^{p}>\frac{\operatorname{dist}\left(a_{\nu}, Y\right)}{\left|a_{\nu}\right|} \geq \frac{1}{\sqrt{n}}\left|\left(\frac{a_{j+1, \nu}}{a_{s, \nu}}, \ldots, \frac{a_{n, \nu}}{a_{s, \nu}}\right)\right| \\
=\frac{1}{\sqrt{n}} \operatorname{dist}\left(u_{\nu}, Y_{s}\right) \geq \frac{C^{\prime \prime}}{\sqrt{n}} \operatorname{dist}\left(u_{\nu}, X_{s} \cap Y_{s}\right)^{p}=\frac{C^{\prime \prime}}{\sqrt{n}}\left|u_{\nu}-w_{\nu}\right|^{p}
\end{gathered}
$$

where $w_{\nu} \in X_{s} \cap Y_{s}$ are points such that $\operatorname{dist}\left(u_{\nu}, X_{s} \cap Y_{s}\right)=\left|u_{\nu}-w_{\nu}\right|$ for $\nu \in \mathbb{N}$.

Let $H_{\infty}=\left\{\left(z_{0}, \ldots, z_{s-1}, z_{s+1}, \ldots, z_{n}\right) \in \mathbb{R}^{n}: z_{0}=0\right\}$. If the set $\{\nu \in$ $\left.\mathbb{N}: w_{\nu} \in H_{\infty}\right\}$ is infinite, then we may assume that $w_{\nu} \in H_{\infty}$ for all $\nu \in \mathbb{N}$. 
Thus $\left|u_{\nu}-w_{\nu}\right| \geq \frac{1}{\left|a_{s, \nu}\right|} \geq \frac{1}{\left|a_{\nu}\right|}$, and by (21),

$$
\frac{1}{\nu} \frac{1}{\left|a_{\nu}\right|}\left(\frac{\operatorname{dist}\left(a_{\nu}, X \cap Y\right)}{1+\left|a_{\nu}\right|^{2}}\right)^{p}>\frac{C^{\prime \prime}}{\sqrt{n}}\left(\frac{1}{\left|a_{\nu}\right|}\right)^{p} \quad \text { for } \quad \nu \in \mathbb{N} \text {. }
$$

Consequently, $\operatorname{dist}\left(a_{\nu}, X \cap Y\right) \geq \nu^{\frac{1}{p}}\left[C^{\prime \prime} / \sqrt{n}\right]^{\frac{1}{p}}\left|a_{\nu}\right|^{1+\frac{1}{p}}$ for $\nu \in \mathbb{N}$, which is impossible. Indeed, $\left|a_{\nu}\right| \rightarrow \infty$, and if $X \cap Y \neq \emptyset$, then for $\nu$ large enough so that $\left|a_{\nu}\right| \geq$ $\operatorname{dist}(0, X \cap Y)$, we have $\operatorname{dist}\left(a_{\nu}, X \cap Y\right) \leq 2\left|a_{\nu}\right|$. If $X \cap Y=\emptyset$, then by convention $\operatorname{dist}\left(a_{\nu}, X \cap Y\right)=1$.

Accordingly, it suffices to consider the case when $w_{\nu} \notin H_{\infty}$ for $\nu \in \mathbb{N}$. In this case for any $\nu \in \mathbb{N}$ there exist $b_{\nu}=\left(b_{1, \nu}, \ldots, b_{n, \nu}\right) \in X \cap Y$ and $\bar{b}_{\nu}=\left(1: b_{1, \nu}: \ldots\right.$ : $\left.b_{n, \nu}\right) \in \bar{X} \cap \bar{Y}$ such that $w_{\nu}=\varphi\left(\bar{b}_{\nu}\right)$. From (21) we have $w_{\nu} \rightarrow u_{0}$ as $\nu \rightarrow \infty$, so $\left|b_{\nu}\right| \rightarrow \infty$, and by (19),

$$
\left|\frac{b_{i, \nu}}{b_{s, \nu}}\right| \leq \frac{1}{2} \quad \text { for } \quad i \neq s \quad \text { and sufficiently large } \quad \nu \in \mathbb{N} .
$$

We may assume that for some $i \in\{1, \ldots, n\},\left|a_{i, \nu}-b_{i, \nu}\right| \geq\left|a_{j, \nu}-b_{j, \nu}\right|$ for all $j=1, \ldots, n$ and $\nu \in \mathbb{N}$. Then we have

$$
\operatorname{dist}\left(a_{\nu}, X \cap Y\right) \leq\left|a_{\nu}-b_{\nu}\right| \leq \sqrt{n}\left|a_{i, \nu}-b_{i, \nu}\right| \quad \text { for } \quad \nu \in \mathbb{N} .
$$

If $i \neq s$, then

$$
\begin{aligned}
\left|u_{\nu}-w_{\nu}\right| & \geq\left|\frac{a_{i, \nu}}{a_{s, \nu}}-\frac{b_{i, \nu}}{b_{s, \nu}}\right| \geq \frac{1}{\left|a_{s, \nu}\right|}\left|a_{i, \nu}-b_{i, \nu}+\frac{b_{i, \nu}}{b_{s, \nu}}\left(b_{s, \nu}-a_{s, \nu}\right)\right| \\
& \geq \frac{1}{\left|a_{\nu}\right|}\left|a_{i, \nu}-b_{i, \nu}\right|-\frac{1}{\left|a_{\nu}\right|}\left|\frac{b_{i, \nu}}{b_{s, \nu}}\right|\left|a_{s, \nu}-b_{s, \nu}\right| .
\end{aligned}
$$

Hence, by (22) we have $\left|u_{\nu}-w_{\nu}\right| \geq \frac{1}{2\left|a_{\nu}\right|}\left|a_{i, \nu}-b_{i, \nu}\right|$; thus, (23) and (21) give

$$
\frac{1}{\nu} \frac{1}{\left|a_{\nu}\right|}\left(\frac{\sqrt{n}\left|a_{s, \nu}-b_{s, \nu}\right|}{1+\left|a_{\nu}\right|^{2}}\right)^{p}>\frac{C^{\prime \prime}}{\sqrt{n}}\left(\frac{\left|a_{s, \nu}-b_{s, \nu}\right|}{2\left|a_{\nu}\right|}\right)^{p} \text { for sufficiently large } \nu \in \mathbb{N},
$$

which is impossible since $\left|a_{\nu}\right| \rightarrow \infty$.

Finally, we consider the last case, $i=s$. In this case we easily obtain $\left|u_{\nu}-w_{\nu}\right| \geq$ $\frac{1}{\left|a_{\nu}\right|\left|b_{\nu}\right|}\left|a_{s, \nu}-b_{s, \nu}\right|$ for $\nu \in \mathbb{N}$. If the sequence $\left(\frac{\left|b_{\nu}\right|}{\left|a_{\nu}\right|}\right)$ is bounded, say by $M>0$, then $\left|u_{\nu}-w_{\nu}\right| \geq \frac{1}{M\left|a_{\nu}\right|^{2}}\left|a_{s, \nu}-b_{s, \nu}\right|$, and thus (23) and (21) give

$$
\frac{1}{\nu} \frac{1}{\left|a_{\nu}\right|}\left(\frac{\sqrt{n}\left|a_{s, \nu}-b_{s, \nu}\right|}{1+\left|a_{\nu}\right|^{2}}\right)^{p}>\frac{C^{\prime \prime}}{\sqrt{n}}\left(\frac{\left|a_{s, \nu}-b_{s, \nu}\right|}{M\left|a_{\nu}\right|^{2}}\right)^{p} \quad \text { for } \quad \nu \in \mathbb{N},
$$

which is impossible. So, the sequence $\left(\frac{\left|b_{\nu}\right|}{\left|a_{\nu}\right|}\right)$ is unbounded and we may assume that $\frac{\left|b_{\nu}\right|}{\left|a_{\nu}\right|} \geq 2$. Then $\frac{\left|a_{\nu}-b_{\nu}\right|}{\left|b_{\nu}\right|} \geq \frac{1}{2}$ for $\nu \in \mathbb{N}$. Hence,

$$
\left|u_{\nu}-w_{\nu}\right| \geq \frac{\left|a_{s, \nu}-b_{s, \nu}\right|}{\left|a_{\nu}\right|\left|b_{\nu}\right|} \geq \frac{1}{\sqrt{n}} \frac{\left|a_{\nu}-b_{\nu}\right|}{\left|a_{\nu}\right|\left|b_{\nu}\right|} \geq \frac{1}{2 \sqrt{n}\left|a_{\nu}\right|} .
$$

Thus, (21) gives

$$
\frac{1}{\nu} \frac{1}{\left|a_{\nu}\right|}\left(\frac{\operatorname{dist}\left(a_{\nu}, X \cap Y\right)}{1+\left|a_{\nu}\right|^{2}}\right)^{p}>\frac{C^{\prime \prime}}{\sqrt{n}}\left(\frac{1}{2 \sqrt{n}\left|a_{\nu}\right|}\right)^{p} \quad \text { for } \quad \nu \in \mathbb{N},
$$

which is impossible since $\left|a_{\nu}\right| \rightarrow \infty$. This proves Claim 3 .

The inequality (15) immediately follows from Claims 3 and 1. This ends the proof. 
Remark 5. If the set of common zeros in $\mathbb{P}^{n}(\mathbb{R})$ of the polynomials $g_{1}, \ldots, g_{k}, h_{1}$, $\ldots, h_{l}$ is finite, then analogously to Theorem 2 we deduce that for some positive constant $C$,

$$
\operatorname{dist}(x, X)+\operatorname{dist}(x, Y) \geq C\left(\frac{\operatorname{dist}(x, X \cap Y)}{1+|x|^{2}}\right)^{\frac{(2 d-1)^{n}+1}{2}} \text { for } \quad x \in \mathbb{R}^{n} .
$$

From Theorem 2 we have

Corollary 10. Let $F=\left(f_{1}, \ldots, f_{m}\right): \mathbb{R}^{n} \rightarrow \mathbb{R}^{m}$ be a polynomial mapping of degree $d \geq 1$. Then for some positive constant $C$,

$$
|F(x)| \geq C\left(\frac{\operatorname{dist}(x, V(F))}{1+|x|^{2}}\right)^{d(6 d-3)^{n-1}} \quad \text { for } \quad x \in \mathbb{R}^{n} .
$$

By the Eojasiewicz exponent at infinity of a mapping $F: \mathbb{R}^{n} \rightarrow \mathbb{R}^{m}$ we mean the supremum of the exponents $\nu$ in the following Łojasiewicz inequality:

$$
|F(x)| \geq C|x|^{\nu} \quad \text { as } \quad|x| \geq R
$$

for some positive constants $C, R$; we denote it by $\mathcal{L}_{\infty}(F)$.

From Corollary 10 we obtain a generalization of the Kollár estimation ( $\mathrm{K}$ ) to the case of polynomial mappings with a compact set of real zeroes.

Corollary 11. Let $F=\left(f_{1}, \ldots, f_{m}\right): \mathbb{R}^{n} \rightarrow \mathbb{R}^{m}$ be a polynomial mapping of degree $d \geq 1$. If $V(F)$ is a compact set, then

$$
\mathcal{L}_{\infty}(F) \geq-d(6 d-3)^{n-1} .
$$

Proof. Since the set $V(F)$ is compact, we have $A|x| \leq \operatorname{dist}(x, V(F)) \leq B|x|$ for some constants $A, B>0$ and sufficiently large $|x|$. Then from Corollary [10 we immediately see that there exist positive constants $C, R$ such that

$$
|F(x)| \geq C|x|^{-d(6 d-3)^{n-1}} \text { for }|x| \geq R .
$$

This gives the assertion.

\section{REFERENCES}

[BM] Edward Bierstone and Pierre D. Milman, Canonical desingularization in characteristic zero by blowing up the maximum strata of a local invariant, Invent. Math. 128 (1997), no. 2, 207-302, DOI 10.1007/s002220050141. MR1440306 (98e:14010)

[Bi] Carles Bivià-Ausina, Injectivity of real polynomial maps and Eojasiewicz exponents at infinity, Math. Z. 257 (2007), no. 4, 745-767, DOI 10.1007/s00209-007-0129-0. MR2342551 (2008g:14122)

[BE] J. Bochnak and S. Łojasiewicz, A converse of the Kuiper-Kuo theorem, Proceedings of Liverpool Singularities-Symposium, I (1969/70), Lecture Notes in Math., Vol. 192, Springer, Berlin, 1971, pp. 254-261. MR0291971 (45 \#1059)

[BR] J. Bochnak and J. J. Risler, Sur les exposants de Łojasiewicz, Comment. Math. Helv. 50 (1975), no. 4, 493-507. MR0404674 (53 \#8474)

[BDLM] Jérôme Bolte, Aris Daniilidis, Olivier Ley, and Laurent Mazet, Characterizations of Eojasiewicz inequalities: subgradient flows, talweg, convexity, Trans. Amer. Math. Soc. 362 (2010), no. 6, 3319-3363, DOI 10.1090/S0002-9947-09-05048-X. MR2592958 (2011j:49023)

[Br] W. Dale Brownawell, Bounds for the degrees in the Nullstellensatz, Ann. of Math. (2) 126 (1987), no. 3, 577-591, DOI 10.2307/1971361. MR916719 (89b:12001)

[C] Jacek Chądzyński, On proper polynomial mappings (English, with Russian summary), Bull. Polish Acad. Sci. Math. 31 (1983), no. 3-4, 115-120. MR742795 (85h:32046a) 
[CK1] Jacek Chądzyński and Tadeusz Krasiński, Sur l'exposant de Eojasiewicz à l'infini pour les applications polynomiales de $\mathbf{C}^{2}$ dans $\mathbf{C}^{2}$ et les composantes des automorphismes polynomiaux de $\mathbf{C}^{2}$ (French, with English and French summaries), C. R. Acad. Sci. Paris Sér. I Math. 315 (1992), no. 13, 1399-1402. MR1199010 (93h:14010)

[CK2] Jacek Chądzyński and Tadeusz Krasiński, The gradient of a polynomial at infinity, Kodai Math. J. 26 (2003), no. 3, 317-339, DOI 10.2996/kmj/1073670612. MR2018725 (2004j:32026)

[Cy] Ewa Cygan, A note on separation of algebraic sets and the Eojasiewicz exponent for polynomial mappings (English, with English and French summaries), Bull. Sci. Math. 129 (2005), no. 2, 139-147, DOI 10.1016/j.bulsci.2004.10.001. MR2123264(2005j:14084)

[CKT] Ewa Cygan, Tadeusz Krasiński, and Piotr Tworzewski, Separation of algebraic sets and the Eojasiewicz exponent of polynomial mappings, Invent. Math. 136 (1999), no. 1, 7587, DOI 10.1007/s002220050304. MR.1681109(2000e:32006)

[DK] Didier D'Acunto and Krzysztof Kurdyka, Explicit bounds for the Eojasiewicz exponent in the gradient inequality for polynomials, Ann. Polon. Math. 87 (2005), 51-61, DOI 10.4064/ap87-0-5. MR2208535 (2006m:32005)

[Ga] A. Gabrièlov, Multiplicities of Pfaffian intersections, and the Eojasiewicz inequality, Selecta Math. (N.S.) 1 (1995), no. 1, 113-127, DOI 10.1007/BF01614074. MR.1327229 (96d:32007)

[Gw] Janusz Gwoździewicz, The Eojasiewicz exponent of an analytic function at an isolated zero, Comment. Math. Helv. 74 (1999), no. 3, 364-375, DOI 10.1007/s000140050094. MR:1710702 (2001d:32037)

[GS] Janusz Gwoździewicz and Stanisław Spodzieja, The Eojasiewicz gradient inequality in a neighbourhood of the fibre, Ann. Polon. Math. 87 (2005), 151-163, DOI 10.4064/ap870-12. MR 2208542 (2007b:14137)

[Ha] Huy Vui Ha, Nombres de Eojasiewicz et singularités à l'infini des polynômes de deux variables complexes (French, with English summary), C. R. Acad. Sci. Paris Sér. I Math. 311 (1990), no. 7, 429-432. MR.1075664(91i:32033)

[J1] Zbigniew Jelonek, On the effective Nullstellensatz, Invent. Math. 162 (2005), no. 1, 1-17, DOI 10.1007/s00222-004-0434-8. MR2198324 (2006k:13057)

[J2] Zbigniew Jelonek, On the Eojasiewicz exponent, Hokkaido Math. J. 35 (2006), no. 2, 471-485. MR2254661(2007d:14109)

[JK] Zbigniew Jelonek and Krzysztof Kurdyka, On asymptotic critical values of a complex polynomial, J. Reine Angew. Math. 565 (2003), 1-11, DOI 10.1515/crll.2003.101. MR2024642 (2005h:32065)

[JKS] Shanyu Ji, János Kollár, and Bernard Shiffman, A global Eojasiewicz inequality for algebraic varieties, Trans. Amer. Math. Soc. 329 (1992), no. 2, 813-818, DOI 10.2307/2153965. MR1046016 (92e:32007)

[K1] János Kollár, Sharp effective Nullstellensatz, J. Amer. Math. Soc. 1 (1988), no. 4, 963975, DOI 10.2307/1990996. MR944576 (89h:12008)

[K2] János Kollár, An effective Eojasiewicz inequality for real polynomials, Period. Math. Hungar. 38 (1999), no. 3, 213-221, DOI 10.1023/A:1004806609074. MR.1756239 (2001f:32009)

[Kui] Nicolaas H. Kuiper, $C^{1}$-equivalence of functions near isolated critical points, Symposium on Infinite-Dimensional Topology (Louisiana State Univ., Baton Rouge, La., 1967), Ann. of Math. Studies, No. 69, Princeton Univ. Press, Princeton, N.J., 1972, pp. 199-218. MR0413161 (54 \#1282)

[Kuo] Tzee Char Kuo, On $C^{0}$-sufficiency of jets of potential functions, Topology 8 (1969), 167-171. MR0238338 (38 \#6614)

[KP] Krzysztof Kurdyka and Adam Parusiński, $\mathbf{w}_{f}$-stratification of subanalytic functions and the Eojasiewicz inequality (English, with English and French summaries), C. R. Acad. Sci. Paris Sér. I Math. 318 (1994), no. 2, 129-133. MR1260324 (95d:32012)

[Kur] Krzysztof Kurdyka, On gradients of functions definable in o-minimal structures (English, with English and French summaries), Ann. Inst. Fourier (Grenoble) 48 (1998), no. 3, 769-783. MR.1644089(2000b:03139)

[KMP] Krzysztof Kurdyka, Tadeusz Mostowski, and Adam Parusiński, Proof of the gradient conjecture of R. Thom, Ann. of Math. (2) 152 (2000), no. 3, 763-792, DOI 10.2307/2661354. MR:1815701(2002d:37028) 
[LT] Monique Lejeune-Jalabert and Bernard Teissier, Clôture intégrale des idéaux et équisingularité (French, with English summary), with an appendix by Jean-Jacques Risler. Ann. Fac. Sci. Toulouse Math. (6) 17 (2008), no. 4, 781-859. MR2499856 (2010i:32026)

[€] S. Łojasiewicz, Une propriété topologique des sous-ensembles analytiques réels (French), Les Équations aux Dérivées Partielles (Paris, 1962), Éditions du Centre National de la Recherche Scientifique, Paris, 1963, pp. 87-89. MR0160856 (28 \#4066)

[Ł1] S. Łojasiewicz, Ensembles semi-analytiques, preprint IHES, 1965.

[Ł2] S. Łojasiewicz, Sur les trajectoires du gradient d'une function analytique, in Geometry Seminars, 1982-1983, Univ. Stud. Bologna, Bologna, 1984, 115-117. MR0771152

[O] Beata Osińska, Extensions of regular mappings and the Eojasiewicz exponent at infinity, Bull. Sci. Math. 135 (2011), no. 2, 215-229, DOI 10.1016/j.bulsci.2010.11.002. MR2773399(2012e:32054)

[Pa] Adam Parusiński, On the bifurcation set of complex polynomial with isolated singularities at infinity, Compositio Math. 97 (1995), no. 3, 369-384. MR1353280 (96i:32038)

[Ph] Pham Tien So'n, The Eojasiewicz exponent of a continuous subanalytic function at an isolated zero, Proc. Amer. Math. Soc. 139 (2011), no. 1, 1-9, DOI 10.1090/S0002-99392010-10683-4. MR2729065(2011k:32039)

[Pł1] A. Płoski, On the growth of proper polynomial mappings, Ann. Polon. Math. 45 (1985), no. 3, 297-309. MR817547 (87d:32047)

[Pł2] A. Płoski, Multiplicity and the Eojasiewicz exponent, Banach Center Publications 20, Warsaw (1988), 353-364. MR1101851

[RS1] Tomasz Rodak and Stanisław Spodzieja, Effective formulas for the Eojasiewicz exponent at infinity, J. Pure Appl. Algebra 213 (2009), no. 9, 1816-1822, DOI 10.1016/j.jpaa.2009.01.016. MR2518180(2010f:14065)

[RS2] Tomasz Rodak and Stanisław Spodzieja, Effective formulas for the local Eojasiewicz exponent, Math. Z. 268 (2011), no. 1-2, 37-44, DOI 10.1007/s00209-009-0659-8. MR.2805423(2012c:32024)

[RS3] Tomasz Rodak and Stanisław Spodzieja, Eojasiewicz exponent near the fibre of a mapping, Proc. Amer. Math. Soc. 139 (2011), no. 4, 1201-1213, DOI 10.1090/S0002-99392010-10623-8. MR:2748414 (2012b:58061)

[Sk] Grzegorz Skalski, On the Eojasiewicz exponent near the fibre of a polynomial, Bull. Pol. Acad. Sci. Math. 52 (2004), no. 3, 231-236, DOI 10.4064/ba52-3-3. MR2127060 (2005m:14118)

[Sp] Stanislaw Spodzieja, The Eojasiewicz exponent of subanalytic sets, Ann. Polon. Math. 87 (2005), 247-263, DOI 10.4064/ap87-0-21. MR2208551 (2006k:32011)

[T] B. Teissier, Variétés polaires. I. Invariants polaires des singularités d'hypersurfaces (French), Invent. Math. 40 (1977), no. 3, 267-292. MR0470246 (57 \#10004)

Laboratoire de Mathematiques (LAMA), Université de Savoie, UMR-5127 de CNRS, 73-376 Le Bourget-du-Lac Cedex, France

E-mail address: Krzysztof.Kurdyka@univ-savoie.fr

Faculty of Mathematics and Computer Science, University of Łódź, S. Banacha 22, 90-238 Łódź, Poland

E-mail address: spodziej@math.uni.lodz.pl 